\title{
TRES ESPECIES NUEVAS DE BURSERA (BURSERACEAE) DE LA REGIÓN COSTERA DEL OCCIDENTE DE MÉXICO*
}

\author{
JERZY RZEDOWSKI \\ Y \\ Graciela Calderón de Rzedowski \\ Instituto de Ecología, A.C. \\ Centro Regional del Bajío \\ Apartado postal 386 \\ 61600 Pátzcuaro, Michoacán
}

\begin{abstract}
RESUMEN
Se describen como nuevas y se ilustran Bursera palaciosii, B. ribana y B. vazquezyanesii, especies aparentemente restringidas en su distribución al cinturón litoral del estado de Jalisco y de algunas áreas adyacentes. La primera está probablemente vinculada con $B$. staphyleoides McVaugh \& Rzed., la segunda es próxima a $B$. laxiflora $S$. Watson, y la tercera parece estar cercanamente emparentada con B. denticulata McVaugh \& Rzed.
\end{abstract}

\section{ABSTRACT}

Bursera palaciosii, B. ribana and B. vazquezyanesii are described as new species and illustrated. All seem to be restricted to the littoral belt of the Mexican state of Jalisco and some adjacent areas. The first one is probably related to $B$. staphyleoides McVaugh \& Rzed., the second one to $B$. laxiflora S. Watson and the third to $B$. denticulata McVaugh \& Rzed.

Exploraciones realizadas en la región próxima al litoral del estado de Jalisco han revelado la existencia de varias especies del género Bursera aún no descritas. Tres de estas plantas se dan a conocer en los siguientes párrafos.

Bursera palaciosii Rzed. \& Calderón sp. nov. Fig. 1.

Arbuscula vel frutex 2-6 m altus ut videtur dioecius glaber; truncus cortice griseo non exfolianti, ramuli pallide straminei; folia trifoliolata vel imparipinnata raro unifoliolata, rhachidi angustissime alata, foliola lateralia (0)2-4 ovata usque elliptica vel lanceolata $1-4.5(6.5) \mathrm{cm}$

\footnotetext{
* Trabajo realizado con apoyo económico del Instituto de Ecología, A.C. (cuenta 902-03), del Consejo Nacional de Ciencia y Tecnología, así como de la Comisión Nacional para el Conocimiento y Uso de la Biodiversidad.
} 


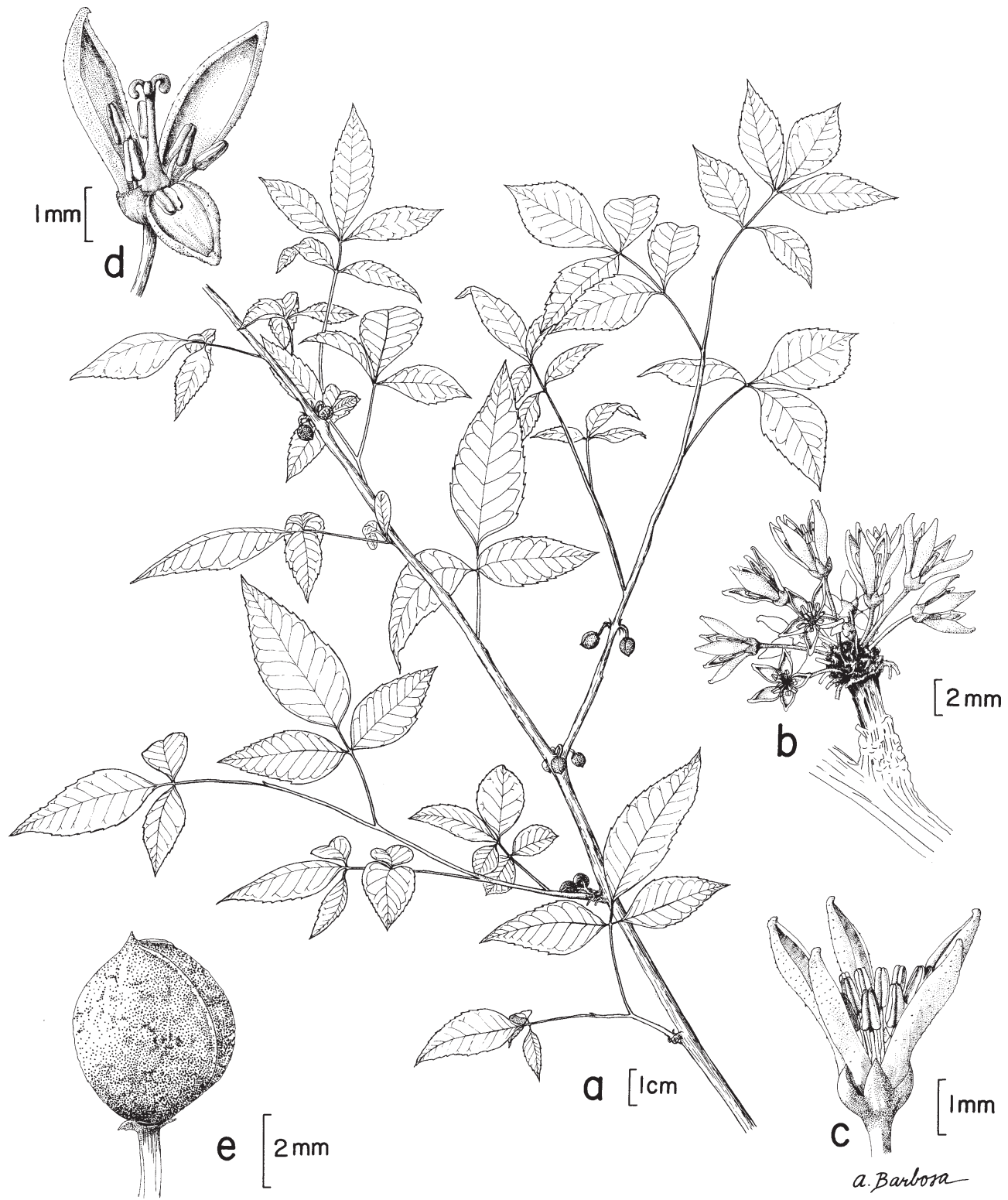

Fig. 1. Bursera palaciosii Rzed. \& Calderón. a. rama con hojas y frutos; b. conjunto de flores masculinas; c. flor masculina; d. flor femenina; e. fruto. Ilustrado por Alfonso Barbosa. 
longa 5-18(25) mm lata, apice acuta vel acuminata, basi cuneata vel rotundata, marginibus plerumque grosse serratis interdum integris vel subintegris, foliolum terminale saepe lateralibus majus, ovatum usque ellipticum, subrhombeum vel obovatum; flores solitarii ad ramulorum brevium extremos aggregati; flores masculi pentameri, calycis lobi anguste triangulares ca. $0.6 \mathrm{~mm}$ longi, petala lanceolato-elliptica albida cucullata ca. $3 \mathrm{~mm}$ longa, stamina 10; flores feminei trimeri masculinorum similes, staminodia 6 , ovarium triloculare, stigmata 3; drupae trivalvatae oblicue subglobosae 4-5(6) $\mathrm{mm}$ diametro conspicue apiculatae, pyrenae pseudoarillo pallido omnino indutae.

Arbolito o arbusto de 2 a $6 \mathrm{~m}$ de alto, aparentemente dioico, caducifolio, glabro, con látex blanquecino; tronco hasta de $25 \mathrm{~cm}$ de diámetro, corteza gris, lisa a tuberculada, no exfoliante, ramillas de color pajizo claro; hojas alternas, mayormente bien espaciadas sobre las ramillas, de 4 a $9 \mathrm{~cm}$ de largo y 3 a $6 \mathrm{~cm}$ de ancho, trifolioladas o imparipinnadas, rara vez alguna unifoliolada, peciolo hasta de $2.5 \mathrm{~cm}$ de largo, raquis muy angostamente alado, peciólulos de los foliolos laterales de 1 a $2.5 \mathrm{~mm}$ de largo, el del foliolo terminal a veces hasta de $7 \mathrm{~mm}$ de largo, foliolos (1)3 ó 5, de forma y tamaño bastante variables, los laterales ovados a elípticos o lanceolados, de 1 a 4.5(6.5) cm de largo, de 5 a 18(2.5) mm de ancho, agudos a acuminados en el ápice, por lo general más bien toscamente aserrados en la mitad distal del margen, a veces enteros o subenteros, cuneados a redondeados y más o menos oblicuos en la base, el foliolo terminal a menudo más grande que los laterales (hasta de $5 \mathrm{~cm}$ de largo y $25 \mathrm{~mm}$ de ancho), variando de ovado a elíptico, subrómbico u obovado, de textura membranácea, un poco más pálidos en el envés que en el haz; flores solitarias, resinosas, por definición axilares, pero por lo general apareciendo antes que las hojas, agrupadas por varias o por muchas en los extremos de ramillas cortas, sobre pedicelos hasta de $4 \mathrm{~mm}$ de largo; las masculinas pentámeras, segmentos del cáliz angostamente triangulares, de ca. $0.6 \mathrm{~mm}$ de largo, pétalos lanceolado-elípticos, blanquecinos a blanco-rosados o blanco-verdosos, cuculados, de ca. $3 \mathrm{~mm}$ de largo, estambres 10, anteras oblongas, de ca. $0.7 \mathrm{~mm}$ de largo, un poco más cortas que los filamentos, gineceo ausente; las femeninas trímeras, similares a las masculinas, pero con los segmentos del cáliz y pétalos un poco más anchos, estaminodios 6 , sus anteras de ca. $0.7 \mathrm{~mm}$ de largo, un poco más largas que los filamentos, ovario trilocular, estigmas 3; drupas trivalvadas, oblicuamente subesféricas, de 4 a 5(6) $\mathrm{mm}$ de diámetro, conspicuamente apiculadas, hueso triangular-subesférico, de 3.5 a $4 \mathrm{~mm}$ de diámetro, totalmente cubierto por el pseudoarilo pálido.

TIPO: México, Jalisco, municipio de La Huerta, Estación de Biología Chamela, cerca de Chamela, alt. $100 \mathrm{~m}$, bosque tropical caducifolio, 26.VII.1982, J. Rzedowski 37882 (holotipo: IEB, isotipos por distribuirse).

Material adicional examinado: Jalisco, municipio de La Huerta, Estación de Biología Chamela, L. A. Pérez J. 1194 (ENCB); ibid., J. A. S. Magallanes 1063 (IEB), 3627 (IEB), 3651 (IEB), 4100 (IEB), 4191 (IEB), 4365 (IEB); ibid., J. Rzedowski 37755 (IEB), 37811 (IEB), 37814 (IEB); ibid., S. H. Bullock 1147 (IEB); ibid., E. J. Lott y A. Solís Magallanes 1026 (IEB); ibid., E. J. Lott 1038 (IEB); Arroyo Tapeixtes (La Mina), km 55 de la carretera Barra de Navidad - Puerto Vallarta, a 4 km al SE de la Estación de Biología, E. J. Lott 2575 (IEB); Rancho Cuixmala. Cumbres 1. Along the road from Station $45(\mathrm{~km} 45$ of the Puerto Vallar- 
ta - Barra de Navidad hwy.) to Cumbres 1, following the Arroyo Limbo. E. J. Lott y B. L. Phillips 3681 (IEB); Rancho Cuixmala. Cumbres 1, Arroyo Cajones. About three km inland from the Puerto Vallarta - Barra de Navidad hwy. E. J. Lott et al. 3797 (IEB).

B. palaciosii pertenece a la sección Bursera en virtud de su ovario trilocular, drupas trivalvadas, así como el escaso desarrollo de los catafilos. Su corteza no exfoliante, sin embargo, marca una notable excepción a la característica común de los miembros de esta sección y con ello muestra similitud con B. paradoxa Guevara \& Rzed. Sin embargo, no debe haber parentesco cercano entre estas dos especies y, al parecer, se trata de otro ejemplo de un proceso de convergencia.

En la morfología de sus hojas $B$. palaciosii se asemeja de manera manifiesta a $B$. staphyleoides McVaugh \& Rzed., que se distribuye en las partes bajas de la porción michoacana de la Cuenca del Balsas, y que con toda probabilidad es la especie filogenéticamente más vinculada. Infortunadamente de esta última no se conocen las flores, por lo que las características diferenciales que pudieron definirse entre ambas son las que se resumen en el Cuadro siguiente:

Cuadro 1. Diferencias morfológicas entre Bursera palaciosii y B. staphyleoides.

\begin{tabular}{|l|l|l|}
\hline & \multicolumn{1}{|c|}{ B. palaciosii } & \multicolumn{1}{c|}{ B. staphyleoides } \\
\hline Corteza externa del tronco & no exfoliante & exfoliante en tiras amarillentas \\
\hline Color de las ramillas & pajizo claro & grisáceo \\
\hline Número de foliolos & (1) 3 ó 5 & 1 ó 3 \\
\hline Peciólulos & de 1 a $2.5 \mathrm{~mm}$ & ausentes \\
\hline Margen de los foliolos & toscamente aserrado a entero & finamente crenado \\
\hline Disposición de las flores & solitarias & $\begin{array}{l}\text { agrupadas en inflorescencias (al } \\
\text { menos algunas de las femeni- } \\
\text { nas) }\end{array}$ \\
\hline Drupa & notablemente apiculada & sin apículo notable \\
\hline
\end{tabular}

McVaugh y Rzedowski (1965, p. 324), en su clasificación tentativa de las especies de Bursera, ubicaron con duda a B. staphyleoides en el grupo 8. Sin embargo, colectas posteriores de la especie en la "tierra caliente" de Michoacán indican que su tronco posee corteza exfoliante en tiras amarillentas, carácter que más bien determina su afinidad con el grupo 11, o sea con B. fagaroides (Kunth) Engl. y especies cercanas, como B. ariensis (Kunth) McVaugh \& Rzed., B. confusa (Rose) Engl. y B. occulta McVaugh \& Rzed. En consecuencia y por extensión procede colocar también a $B$. palaciosii en este mismo conjunto. 
En su carácter de flores solitarias, el taxon que se describe como nuevo no solamente converge también con el grupo de $B$. fagaroides, sino que representa el extremo de la tendencia de la reducción del tamaño y de la complejidad de la inflorescencia.

De lo anterior cabe deducir que, entre sus congéneres próximos, B. palaciosii debe considerarse como una especie muy evolucionada en cuanto se refiere a la supresión del carácter de corteza exfoliante, así como a la reducción de la inflorescencia y del número de foliolos. Cabe notar que en esta última tendencia $B$. staphyleoides ha avanzado aún más lejos.

B. palaciosii sólo se conoce hasta la fecha de los terrenos de la Estación de Biología Chamela (de la Universidad Nacional Autónoma de México) y de algunos lugares adyacentes, donde se manifiesta como componente más bien esporádico a poco abundante del bosque tropical caducifolio, que prospera entre 50 y $500 \mathrm{~m}$ de altitud en laderas relativamente próximas al litoral pacífico. Florece de mayo a junio.

El nombre de la especie honra la memoria del destacado palinólogo mexicano Rodolfo Palacios Chávez, fallecido en 1999. El doctor Palacios publicó varios artículos relacionados con la morfología del polen de representantes de Burseraceae, sin menoscabo de otros referentes a la flora palinológica de la Estación Biológica de Chamela.

Bursera ribana Rzed. \& Calderón sp. nov. Fig. 2.

Arbuscula vel frutex 2-6 m altus ut videtur dioecius; truncus cortice griseo non exfolianti, ramuli atrorubri glabri; foliorum rosulae juventute cataphyllis exterioribus mature deciduis cinctae, folia imparipinnata usque bipinnata, rhachidi rhachillisque conspicue alatis, foliola primaria (5)9-11(15) plerumque ovata usque obovata (6)10-20(25) mm longa 6-16 mm lata (foliolum terminale plerumque lateralibus valde majus), apice acuta usque fere truncata, basi generaliter cuneata, marginibus grosse profundeque serrata, supra puberula vel fere glabra, subtus puberula vel hirsuta saepe glabrescentia, cartacea; inflorescentiae racemosopaniculatae; flores masculi tetrameri puberuli, calycis lobi late triangulares ca. $0.5 \mathrm{~mm}$ longi, petala alba oblanceolato-elliptica ca. $2 \mathrm{~mm}$ longa, stamina 8; flores feminei masculorum similes, staminodia 8, ovarium biloculare, stigmata 2; drupae obovoideae 5-6 mm longae, pyrenae biconvexae pseudoarillo aurantiaco per $2 / 3$ partibus inferis indutae.

Arbolito o arbusto de 2 a $6 \mathrm{~m}$ de alto, aparentemente dioico, caducifolio, con oleoresina de aroma agradable; tronco hasta de $30 \mathrm{~cm}$ de diámetro, corteza gris, no exfoliante, ramillas rojas oscuras, glabras; hojas concentradas en rosetas en los extremos de ramillas cortas, originadas en años pasados, precedidas en su aparición por un conjunto de catafilos lineares a oblongos o suborbiculares, de 2 a $6 \mathrm{~mm}$ de largo y 1 a $2 \mathrm{~mm}$ de ancho, densamente pubescentes, de consistencia coriácea, muy pronto caedizos, hojas imparipinnadas a parcial o casi totalmente bipinnadas (estas últimas sólo se observan en algunas ramillas jóvenes estériles), hasta de $11 \mathrm{~cm}$ de largo y $8 \mathrm{~cm}$ de ancho, peciolo hasta de $1.5 \mathrm{~cm}$ de largo, raquis (y raquillas) con alas manifiestas, de 1 a $3 \mathrm{~mm}$ de ancho de cada lado, con el margen entero, incrementando su anchura hacia la parte distal del intersticio, peciólulos por lo general ausentes, pero a veces hasta de $2.5 \mathrm{~mm}$ de largo y ligeramente alados, foliolos (en las hojas una vez pinnadas) (5)9 a 11(15), por lo general ovados a obovados, variando a oblanceolados, subrómbicos o suborbiculares, de (6)10 a 


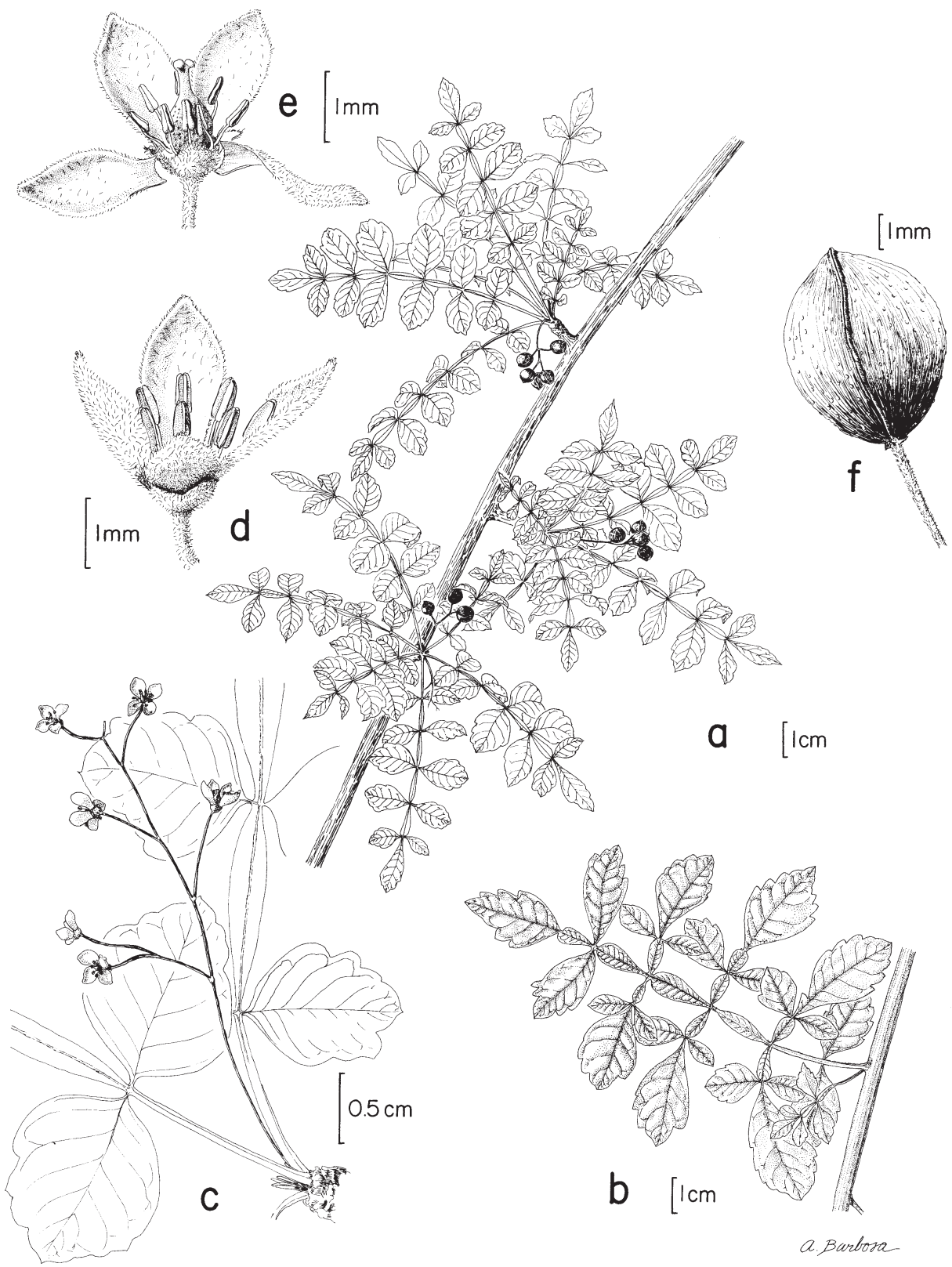

Fig. 2. Bursera ribana Rzed. \& Calderón. a. rama con hojas y frutos; b. hoja casi totalmente bipinnada, c. inflorescencia masculina; d. flor masculina; e. flor femenina; f. fruto. llustrado por Alfonso Barbosa. 
Rzedowski y Calderón de Rzedowski: Tres Especies Nuevas de Bursera de la Región Costera

20(25) $\mathrm{mm}$ de largo y 6 a $16 \mathrm{~mm}$ de ancho, el terminal a menudo más grande que los laterales, ápice agudo a casi truncado, margen tosca y profundamente serrado con (1)2 a 5(6) dientes de cada lado, base por lo general cuneada, pero variando a truncada y abruptamente cuneada, a menudo oblicua, sobre todo en los foliolos laterales, finamente pubérulos a casi glabros en el haz, en el envés pubérulos a hirsutos, principalmente a lo largo de las nervaduras, con la edad más o menos glabrescentes, verdes oscuros y algo brillantes en el haz, un poco más pálidos en el envés, de textura cartácea, los foliolos de las hojas bipinnadas 3 a 5 por pinna, los laterales por lo general muy pequeños, sobre todo en comparación con el terminal que suele ser mucho más grande; inflorescencias axilares, racimoso-paniculadas, a menudo presentes al mismo tiempo que las hojas tiernas, bracteadas, sus ejes finos, pubérulos; flores de ambos sexos tetrámeras; inflorescencias masculinas hasta de $8 \mathrm{~cm}$ de largo, pedicelos hasta de 6(7) $\mathrm{mm}$ de largo, cáliz dividido casi hasta la base, sus lóbulos anchamente triangulares, de ca. $0.5 \mathrm{~mm}$ de largo, pubérulos, pétalos blancos, oblanceolado-elípticos, de ca. $2 \mathrm{~mm}$ de largo, pubérulos a hirsútulos, estambres 8 , anteras oblongas, de ca. $0.6 \mathrm{~mm}$ de largo, un poco más cortas que los filamentos, gineceo diminuto o ausente; inflorescencias femeninas hasta de $6 \mathrm{~cm}$ de largo, pedicelos hasta de $15 \mathrm{~mm}$ de largo, flores similares a las masculinas, estaminodios 8 , anteras de ca. $0.4 \mathrm{~mm}$ de largo, ovario bilocular, estigmas 2; drupas obovoides, de 5 a $6 \mathrm{~mm}$ de largo, hueso biconvexo, de ca. $4 \mathrm{~mm}$ de largo, cubierto en sus 2/3 partes inferiores por un pseudoarilo anaranjado, la parte expuesta negra.

TIPO: México, Jalisco, municipio de Tomatlán, $6 \mathrm{~km}$ al $\mathrm{S}$ de Tomatlán, sobre la carretera a La Cumbre, alt. $50 \mathrm{~m}$, bosque tropical caducifolio sobre ladera granítica, 27.VII.1982, J. Rzedowski 37886 (holotipo en IEB, isotipos por distribuirse).

Material adicional examinado: Jalisco, municipio de Tomatlán, $5 \mathrm{~km}$ al O de Tomatlán, camino a la carretera Barra de Navidad - Pto. Vallarta, J. A. S. Magallanes 3658 (IEB); $4 \mathrm{~km}$ al W de Tomatlán, sobre el camino que entronca a la carretera costera, J. Rzedowski y R. McVaugh 1348 (IEB); $6 \mathrm{~km}$ al S de Tomatlán, sobre la carretera a La Cumbre, J. Rzedowski 37885 (IEB); municipio de La Huerta, Estación de Biología de Chamela, L. A. Pérez J. 1064 (IEB); cerro Huehuentón, 20-25 km al E de Chamela, J. Rzedowski y R. McVaugh 1366 (IEB); 2 km al S de La Huerta, por la carretera a Barra de Navidad, 104'40' W, 19²8' N, S. Carbajal H. y A. Casillas 2388 (IEB); ibid., J. A. Pérez de la Rosa 170 (IEB); ibid., J. Rzedowski 37816 (IEB). Michoacán: municipio de Aquila, $3 \mathrm{~km}$ al W de Cruz de Cachán, B. Guerrero C. 1009 (IEB).

A raíz de su ovario bilocular, corteza no exfoliante y presencia de catafilos relativamente bien desarrollados, $B$. ribana se ubica en la sección Bullockia y en la clasificación de McVaugh y Rzedowski (op. cit., p. 323) parece acomodarse mejor en el grupo 1, al lado de B. bipinnata (Moc. \& Sessé ex DC.) Engl.

En efecto, la especie nueva se acerca a esta última en el hecho de que algunas de sus hojas son al menos parcialmente bipinnadas, además de mostrar semejanza en la textura de los foliolos, en la fenología de la floración, en la organización de la inflorescencia, así como en la forma y en el tamaño del fruto y del hueso.

Tanto Bullock (1936), como McVaugh y Rzedowski (op. cit.) y también Rzedowski y Guevara-Féfer (1992) hicieron ver que B. bipinnata se cruza con cierta frecuencia con 
otros miembros de la sección Bullockia, en particular con B. copallifera (Moc. \& Sessé ex DC.) Bullock, B. cuneata (Schltdl.) Engl., B. excelsa (Kunth) Engl., B. palmeri S. Watson y B. tomentosa (Jacq.) Triana \& Planch. Tales híbridos aislados a menudo se han identificado como $B$. diversifolia Rose, pero al parecer este nombre no corresponde en realidad a ninguna especie, sino que colectivamente han quedado asignadas ahí muchas plantas diferentes.

Es factible que $B$. ribana (o su antecesor) haya derivado de un antiguo cruzamiento de $B$. bipinnata con alguna otra especie de posible afinidad con $B$. aloexylon (Schiede ex Schltdl.) Engl. o con B. glabrifolia (Kunth) Engl. En la actualidad no convive con ninguno de estos posibles progenitores y su vinculación filogenética se dirige más bien hacia un grupo de especies distribuidas a lo largo del litoral pacífico, la más cercana de las cuales probablemente es $B$. laxiflora $\mathrm{S}$. Watson.

$B$. laxiflora se distribuye fundamentalmente en Sonora, con extensiones a Sinaloa, Baja California Sur y Chihuahua (Johnson, 1992) y coincide en muchos caracteres con $B$. ribana, incluyendo la tendencia a la forma bipinnada de las hojas. En el cuadro siguiente se resumen las principales diferencias entre estos dos taxa, tomando también como referencia a $B$. bipinnata:

Cuadro 2. Principales diferencias entre Bursera laxiflora, B. ribana y B. bipinnata.

\begin{tabular}{|l|l|l|l|}
\hline & \multicolumn{1}{|c|}{ B. laxiflora } & \multicolumn{1}{c|}{ B. ribana } & \multicolumn{1}{c|}{ B. bipinnata } \\
\hline $\begin{array}{l}\text { Raquis y raquillas de } \\
\text { la hoja }\end{array}$ & angostamente alados & anchamente alados & angostamente alados \\
\hline $\begin{array}{l}\text { Número de foliolos pri- } \\
\text { marios }\end{array}$ & 5 a 15 & 5 a 15 & 15 a 35 \\
\hline Ancho del foliolo & 3 a $8(12) \mathrm{mm}$ & 6 a $16 \mathrm{~mm}$ & 2 a $5(9) \mathrm{mm}$ \\
\hline $\begin{array}{l}\text { Dientes de cada lado } \\
\text { del foliolo }\end{array}$ & 1 a $3(4)$ & $(1) 2$ a $5(6)$ & 0 a 1 \\
\hline Pubescencia de la flor & ausente & presente & presente \\
\hline $\begin{array}{l}\text { Proporción entre el largo } \\
\text { de los segmentos del } \\
\text { cáliz y el de los péta- } \\
\text { los }\end{array}$ & ca. $1 / 2$ & ca. $1 / 4$ & ca. 1 \\
\hline
\end{tabular}

De la misma afinidad filética resulta ser también B. filicifolia Brandegee, restringida en su distribución al sector meridional de Baja California Sur, y que algunos autores prefieren considerar como variedad de B. laxiflora. Esta especie se caracteriza principalmente por sus ramillas de color pálido y por la pubescencia mucho más densa de las hojas. 
Toledo Manzur (1982, pp. 94-95) consideró bajo el nombre de B. aff. diversifolia una planta procedente de la costa de Guerrero y de Michoacán, que probablemente también pertenece al mismo conjunto. Con base en ejemplares estériles, el mencionado autor la describió como arbusto espinoso con corteza exfoliante en rígidas tiras verticales, llevando hojas con 6 pares de foliolos primarios, los simples ovados, de 1.5 a $3.5 \mathrm{~cm}$ de largo y 1.4 a $1.6 \mathrm{~cm}$ de ancho.

Otro taxon aún sin describir, con toda verosimilitud vinculado con $B$. laxiflora y con B. ribana, se ha colectado en los alrededores de Tehuantepec y de Salina Cruz, en el sureste de Oaxaca. Es un árbol de 6 a $8 \mathrm{~m}$ de altura, con hojas de 7 a 15 foliolos primarios, que miden hasta cerca de $4 \mathrm{~cm}$ de largo y $2 \mathrm{~cm}$ de ancho, con puberulencia muy densa tanto en los órganos foliares como en los florales.

Estas cinco entidades constituyen al parecer un interesante ejemplo de un grupo de especies vicariantes, que evolucionaron y se dispersaron a lo largo del litoral pacífico de México a partir de un antecesor común.

Fuera del ámbito de parentesco cercano, cabe indicar que en la forma de sus hojas $B$. ribana se asemeja a, y podría confundirse a veces con $B$. aloexylon, $B$. glabrifolia y $B$. mirandae Toledo. Las dos primeras se separan por sus flores y frutos más grandes; la tercera difiere en el escaso o nulo desarrollo de las alas del raquis foliar.

La especie nueva se distribuye a lo largo de la costa de Jalisco, muy probablemente también de Colima, llegando hasta el extremo SW de Michoacán. Se ha colectado entre 50 y $450 \mathrm{~m}$ de altitud, en el bosque tropical caducifolio, así como en el subcaducifolio. Florece de junio a agosto.

El nombre de este taxon se consagra a la memoria del finado Ramón Riba y Nava Esparza, ameritado botánico mexicano, particularmente dedicado al estudio de las pteridofitas.

Bursera vazquezyanesii Rzed. \& Calderón sp. nov. Fig. 3.

Arbuscula 4-5 m alta ut videtur dioecia glabra; truncus cortice exteriore papyraceo aurantiaco exfolianti; folia imparipinnata, rhachidi anguste alata, foliola (3)7-9 lanceolatosubrhombea 2.5-6.5 cm longa 7-18 mm lata, apice acuminato-attenuata, basi cuneata vel rotundata, margine serrata; inflorescentiae masculae cymoso-paniculatae, flores masculi pentameri, calycis lobi late triangulares ca. $0.5 \mathrm{~mm}$ longi, petala lanceolato-ovata rubella cucullata ca. $3 \mathrm{~mm}$ longa, stamina 10; inflorescentiae femineae (1)3(5)-florae, flores feminei plerumque tetrameri, masculinorum similes sed petalis $2-2.5 \mathrm{~mm}$ longis, staminodia generaliter 8, ovarium triloculare, stigmata 3; drupae trivalvatae oblicue subglobosae 3-4 mm diametro, pyrenae triangulari-subglobosae pseudoarillo pallido omnino indutae.

Arbolito de 4 a $5 \mathrm{~m}$ de alto, aparentemente dioico, caducifolio, resinoso, glabro; tronco hasta de $25 \mathrm{~cm}$ de diámetro, de corteza lisa, anaranjada, exfoliándose en grandes tiras delgadas, papiráceas; hojas mayormente aglomeradas en forma de rosetas sobre extremos de ramillas cortas originadas en años pasados, o bien, alternas y esparcidas sobre ramillas vigorosas jóvenes, hasta de $15 \mathrm{~cm}$ de largo y $9 \mathrm{~cm}$ de ancho, imparipinnadas, peciolo hasta de $5 \mathrm{~cm}$ de largo, canaliculado del lado adaxial, foliolos (3)7 a 9, raquis muy estrecha y uniformemente alado, peciólulos por lo general de $1 \mathrm{~mm}$ o menos de largo, el del foliolo 


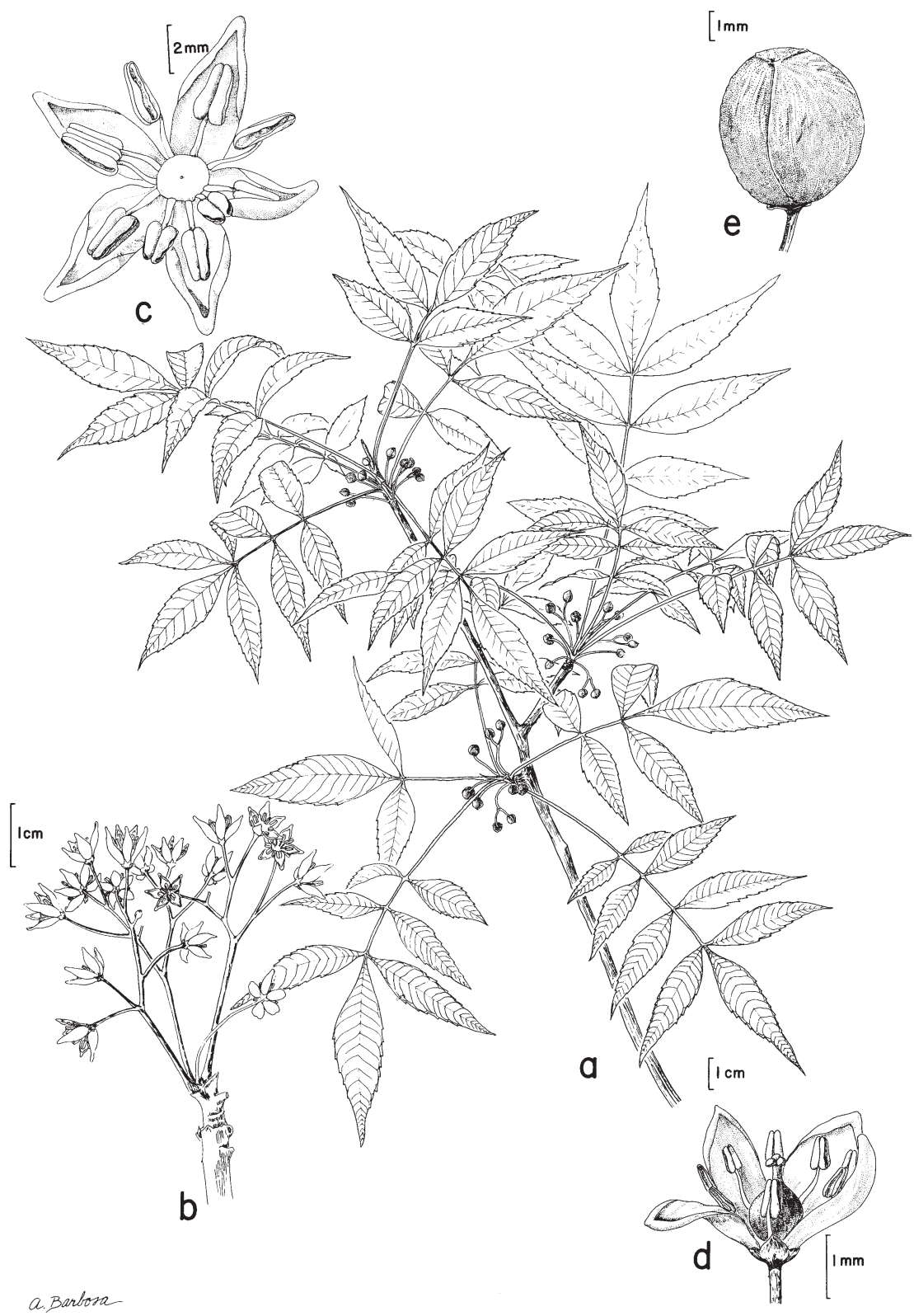

Fig. 3. Bursera vazquezyanesii Rzed. \& Calderón. a. rama con hojas y frutos; b. conjunto de inflorescencias masculinas; c. flor masculina; d. flor femenina; e. fruto. Ilustrado por Alfonso Barbosa. 
Rzedowski y Calderón de Rzedowski: Tres Especies Nuevas de Bursera de la Región Costera

terminal a veces hasta de $7 \mathrm{~mm}$ de largo, foliolos lanceolado-subrómbicos, de 2.5 a $6.5 \mathrm{~cm}$ de largo, de 7 a $18 \mathrm{~mm}$ de ancho, por lo común disminuyendo de tamaño hacia la base de la hoja, ápice acuminado-atenuado, margen serrado con 5 a 15 dientes de cada lado, quedando enteros el acumen y la porción proximal, base cuneada a redondeada, por lo general oblicua en los foliolos laterales, de textura membranácea, algo más pálidos en el envés; inflorescencias por definición axilares, pero con frecuencia dispuestas en grupos sobre extremos de ramillas cortas originadas en años pasados, apareciendo poco antes que las hojas, por lo general muy resinosas; las masculinas cimoso-paniculadas, hasta de $3 \mathrm{~cm}$ de largo, con pedúnculos hasta de $2 \mathrm{~cm}$ y pedicelos hasta de $1 \mathrm{~cm}$ de largo, flores masculinas pentámeras, lóbulos del cáliz anchamente triangulares, de ca. $0.5 \mathrm{~mm}$ de largo, rojizos, pétalos lanceolado-ovados, rojizos, cuculados, de ca. $3 \mathrm{~mm}$ de largo, agudos en el ápice, estambres 10, anteras oblongas, de ca. $1 \mathrm{~mm}$ de largo, un poco más largas que los filamentos, gineceo ausente; inflorescencias femeninas (1)3(5)-floras, hasta de $2 \mathrm{~cm}$ de largo, con pedicelos hasta de $6 \mathrm{~mm}$ de largo, flores por lo general tetrámeras, similares a las masculinas, pero con pétalos de 2 a $2.5 \mathrm{~mm}$ de largo, estaminodios comúnmente 8, sus anteras de ca. $0.5 \mathrm{~mm}$ de largo, ovario trilocular, estigmas 3; drupas trivalvadas, oblicuamente subglobosas, de 3 a $4 \mathrm{~mm}$ de diámetro, huesos de ca. $3 \mathrm{~mm}$ de diámetro, triangular-subglobosos, envueltos totalmente por un pseudoarilo pálido.

TIPO: México, Jalisco, municipio de Cabo Corrientes, $14 \mathrm{~km}$ al W de El Tuito, sobre el camino a Llano Grande de Ipala, alt. 500 m, bosque de Quercus sobre laderas graníticas, 27.VII.1982, J. Rzedowski 37888 (holotipo: IEB, isotipos por distribuirse).

Material adicional examinado: Jalisco, municipio de Cabo Corrientes, cerca de Zicatán, 8 km al W de El Tuito, J. Rzedowski 37759 (IEB), 37760 (IEB); 14 km al W de El Tuito, sobre la carretera a Ipala, J. Rzedowski 37821 (IEB), 37822 (IEB).

En virtud de su ovario tricarpelar, de su corteza exfoliante, así como de la falta de catafilos bien desarrollados, la especie nueva pertenece a la sección Bursera y encuentra buen acomodo en el grupo 10 de la clasificación preliminar de McVaugh y Rzedowski (1965, p. 324), al lado de B. denticulata McVaugh \& Rzed., B. fragilis S. Watson, B. kerberi Engl., B. lancifolia (Schltdl.) Engl. y B. multijuga Engl.

En el aspecto general de sus hojas $B$. vazquezyanesii se asemeja mucho a $B$. fragilis (distribuida de Sonora y Chihuahua a Sinaloa y Durango), así como a B. lancifolia (conocida de Morelos, Guerrero, Puebla y Oaxaca, y en forma disyunta de Querétaro). Algunos autores, como Bullock (op. cit.) y Johnson (op. cit.) han considerado a estas dos últimas como pertenecientes a una sola especie, pero las diferencias en la morfología de los frutos, en la nerviación de las hojas y en el color de la corteza indican que se trata más probablemente de dos entidades separadas. En términos de distribución geográfica $B$. vazquezyanesii se ubica en medio de $B$. fragilis y de $B$. lancifolia y el color anaranjado de la corteza de su tronco es intermedio entre el amarillento de la especie septentrional y el rojo de la meridional. Sin embargo, el tamaño notablemente más pequeño de los frutos, la presencia de peciólulos cortos, así como detalles de la estructura de la inflorescencia y de las flores de la planta nueva de Jalisco, son indicativos de que su parentesco real más cercano debe buscarse tal vez en otra dirección. 
Es probable que $B$. vazquezyanesii presente vínculos filogenéticos estrechos con B. denticulata, conocida del oeste de Jalisco al oeste de Guerrero. Esta última tiene hojas con 7 a 11 foliolos elípticos a lanceolados, de 1.5 a 3(4.5) cm de largo, de ápice agudo pero que varía de obtuso a acuminado y de márgenes finamente crenado-serrados, la estructura de sus inflorescencias, flores y frutos es muy similar a la de la especie que se describe, aunque estos últimos son un poco más grandes en $B$. denticulata, cuya corteza también es algo diferente, pues se ha observado de color rojo.

Por otra parte, como lo señalaron McVaugh y Rzedowski (op. cit., pp. 364-365), en las áreas de simpatría de $B$. multijuga y de $B$. kerberi, e inclusive en donde una de estas dos especies convive con miembros del complejo de B. fagaroides (Kunth) Engl., se encuentran ocasionalmente individuos de origen híbrido con hojas similares a las de $B$. fragilis y $B$. lancifolia. En el mismo trabajo se supone que la entidad descrita como $B$. confusa Rose puede haberse originado por cruzamiento entre $B$. multijuga y algún componente del grupo fagaroides.

Las hojas de $B$. vazquezyanesii en su morfología pueden considerarse como de forma intermedia entre las de $B$. kerberi y de $B$. multijuga y sería razonable postular que la especie nueva tiene su último origen en una hibridación. En la actualidad no cohabita con ninguno de estos probables progenitores y difiere de ambos en la corteza que no es roja brillante y en el tamaño de los frutos que es aún algo menor.

De lo anterior cabe deducir que en el proceso evolutivo de este grupo de especies de Bursera deben haber jugado un papel importante la hibridación, así como la convergencia morfológica y en tal virtud nos encontramos ante un proceso filogenético que a menudo puede asumir la forma reticular.

B. vazquezyanesii parece estar restringida en su distribución a un pequeño sector del extremo occidental del estado de Jalisco, donde habita a unos $500 \mathrm{~m}$ de altitud, en un encinar que desciende hasta lugares relativamente cercanos al litoral pacífico.

El epíteto específico honra la memoria del recientemente desaparecido botánico mexicano Carlos Vázquez Yanes, renombrado sobre todo por sus contribuciones al conocimiento de la ecofisiología de semillas de un gran número de plantas de nuestro país.

\section{AGRADECIMIENTOS}

Se dan las gracias al Biól. J. Arturo Solís Magallanes, quien ayudó en forma entusiasta y substancial en las colectas realizadas en la Estación de Biología Chamela.

\section{LITERATURA CITADA}

Bullock, A. A. 1936. Notes on the Mexican species of the genus Bursera. Bull. Misc. Inf. Kew 1936: 346-387.

Johnson, M. B. 1992. The genus Bursera (Burseraceae) in Sonora, Mexico and Arizona, U.S.A. Desert Plants 10: 126-143.

McVaugh, R. y J. Rzedowski. 1965. Synopsis of the genus Bursera L. in western Mexico, with notes on the material of Bursera collected by Sessé \& Mociño. Kew Bull. 18: 317-382. 
Rzedowski y Calderón de Rzedowski: Tres Especies Nuevas de Bursera de la Región Costera

Rzedowski, J. y F. Guevara-Féfer. 1992. Familia Burseraceae. Fascículo 3. Flora del Bajío y de Regiones Adyacentes. Instituto de Ecología, A.C. Pátzcuaro, Mich. 46 pp.

Toledo Manzur, C. A. 1982. El género Bursera (Burseraceae) en el estado de Guerrero (México). Tesis de licenciatura. Facultad de Ciencias. Universidad Nacional Autónoma de México. México, D.F. $182 \mathrm{pp}$.

Recibido en diciembre de 1999.

Aceptado en marzo de 2000. 